\title{
SIRT1 Activation Attenuates Microglia Mediated Synaptic Engulfment in Postoperative Cognitive Dysfunction
}

\section{Yi Sun}

Beijing Chao-Yang Hospital

\section{Yuzhu Wang}

Beijing Chao-Yang Hospital

Fan Ye

Beijing Chao-Yang Hospital

Dandan Lin

Beijing Chao-Yang Hospital

\section{Hui Shi}

Beijing Chao-Yang Hospital: Beijing Chaoyang Hospital

\section{Yan Zhang}

Peking University

Anshi Wu ( $\nabla$ wuanshi1965@163.com )

Beijing Chao-Yang Hospital https://orcid.org/0000-0003-2353-2184

\section{Changwei Wei}

Beijing Chao-Yang Hospital

\section{Research Article}

Keywords: SIRT1, microglia, engulfment, postoperative cognitive dysfunction

Posted Date: February 19th, 2021

DOI: https://doi.org/10.21203/rs.3.rs-209946/v1

License: (1) (1) This work is licensed under a Creative Commons Attribution 4.0 International License. Read Full License 


\section{Abstract}

\section{Background}

Postoperative cognitive dysfunction (POCD) is a debilitating neurological complication in surgical patients. Current studies mainly focus on microglia activation, however, less is known about the mechanism underlying neuronal synaptic changes involved in microglia activation. Recent studies indicate that silent information regulator 1 (SIRT1) plays critical roles in different neurological disorders, and is involved in microglia activation. Herein, we evaluated the effects of SIRT1 activation on POCD.

Methods

Exploratory laparotomy were employed on mice (12-14 month) under sevoflurane anesthesia to establish model of POCD. Transcriptional changes in hippocampus after anesthesia and surgery were evaluated by RNA sequencing. SIRT1 expression were verified by Western Blot. Mice were treated with SIRT1 agonist SRT1720 or vehicle after surgery. Changes in microglia morphology, microglial phagocytosis, as well as dystrophic neurites and dendritic spine density were determined. Cognitive functions were evaluated by $Y$ maze and Morris water maze.

Results

SIRT1 expression levels were downregulated in anesthesia and surgery group. Anesthesia and surgery lead to microglia morphology alteration, enhanced synaptic engulfment, dendritic spine loss, and cognitive deficits in our mice model, all of which were alleviated by SRT1720 administration.

\section{Conclusion}

Our study unveils one of the roles of SIRT1 in POCD pathogenesis. SIRT1 activation may represent a therapeutic strategy for prevention and treatment of POCD.

\section{Introduction}

Postoperative cognitive dysfunction (POCD) is a frequent complication of the central nervous system in surgical patients, which is typically characterized by memory decline, inattention, and impaired orientation following surgery [1]. The clinical symptoms can last for months or even years, resulting in adverse conditions (including delayed postoperative recovery, prolonged hospitalization and increased mortality) [2]. Although it is thought that neuroinflammation, oxidative stress, amyloid beta deposition and hyperphosphorylation of tau protein may involve in POCD pathogenesis $[2,3]$, mechanisms of POCD remain largely obscure.

The vast majority of research focused at alleviating neuroinflammation in POCD, and the common feature among these studies is microglia activation [4-6]. Microglia originate in the yolk sac and migrate in the brain during embryonic stage [7]. Microglia are considered as brain-resident macrophages, which 
are isolated from the rest of body by blood brain barrier [8]. In brain development, homeostatic microglia are key regulator of synaptic pruning and synaptic refinement [9]. Besides, microglia serve a surveillance role in eliminating apoptotic debris and invading pathogens in the mature brain [10]. However, abnormal microglia activation has detrimental effect on synaptic connectivity and synaptic functions in various neurological disorders [11]. From our previous study, microglia activation and synapse lose are observed in the hippocampus of POCD mouse model [12].

Silent information regulator 1 (SIRT1) is a nicotinamide adenine dinucleotide $\left(\mathrm{NAD}^{+}\right)$-dependent class III histone deacetylase of the sirtuin family, presented in a wide range of tissues including the brain [13]. SIRT1 binds to and deacetylases various targets such as nuclear factor-kappa B (NF-KB), p65, forkhead box $\mathrm{O}$ (FOXO), peroxisome proliferator-activated receptor alpha (PPARa) and PPAR-gamma co-activator 1alpha (PGC-1a), modulating a variety of cellular processes [14]. SIRT1 is recognized to have neuroprotective properties in neurodegenerative diseases and psychiatric disorders [15,16]. However, far less is known regarding the molecular roles of SIRT1 in the development of POCD.

In current study, we demonstrate that anesthesia and surgery induces SIRT1 dysfunction and microglia activation in hippocampus. SIRT1 agonist SRT1720 protected against synaptic engulfment by microglia. Together, our data provide evidence that abnormal microglia activation mediates synaptic engulfment in POCD. SIRT1 activation may become a promising therapeutic approach to prevent synapse loss and preserve cognitive function in POCD.

\section{Materials And Methods}

\section{Animals}

Wild-type C57BL/6 mice (12-14 month of age) were used for all experiment. Mice were purchased from Vital River Laboratory Animal Technologies (Beijing, China) at 9 month-old. All mice were housed on a 12hour light/dark cycle with ad libitum access to water and food. All experimental procedures were approved by Institutional Animal Care and Use Committee at Capital Medical University (protocol AEEI2020-117). The schematic of the experimental design is presented in Fig.1

\section{Establishment of POCD mice model}

Mice received exploratory laparotomy as previous described [17]. Mice were anesthetized by $5 \%$ sevoflurane for three minutes in a chamber. After induction, we moved mice out of chamber and performed surgical procedure under $2 \%$ sevoflurane. A midline abdominal incision approximately $3 \mathrm{~cm}$ was made to expose surgical field. Liver, stomach, spleen, kidney, intestine and bladder were explored in sequence. The muscle fascia and skin were closed with 5-0 nylon sutures. Procedure lasted around 15 min. $0.2 \%$ ropivacaine was injected on incision subcutaneously for postoperative analgesia. Sevoflurane anesthesia were terminated after the operation, and mice were returned to their cages. Temperature was maintained at $37^{\circ} \mathrm{C}$ during surgery using a heating pad. Mice that served as control, did not underwent any anesthesia or surgery. 


\section{RNA-seq and bioinformatic analysis}

Total RNA was extracted from hippocampi using Trizol (Tiangen, Beijing, China). Complementary DNA was synthesized with DNA polymerase I and RNaseH. Products were purified by polymerase chain reaction to conduct the library, and then were quantified using Agilent 2100 Bioanalyzer (Agilent Technologies, Santa Clara, CA, USA). Sequencing were performed using Illumina HiSeq sequencer (Illumina).

Data were aligned using HISAT2 (Johns Hopkins University) with default parameters [18]. HTseq was used for quantification and R (version 5.2.0) was used for bioinformatics analysis. DESeq2 (version 1.30.0) was used for differential expression analysis. ClusterProfile (version 3.10) was used for Gene Ontology (GO) and network analysis. Gene set enrichment analysis (GSEA) was performed using MSigDB (version 7.0).

\section{Drug administration}

SRT1720 (S1129, Selleck, Houston, TX, USA), a SIRT1 agonist, was dissolved in dimethyl sulfoxide (DMSO) at a concentration of $40 \mathrm{mg} / \mathrm{ml}$ and stored at $-20^{\circ} \mathrm{C}$. SRT 1720 was further diluted by saline solution in use. Mice were injected intraperitoneally (i.p.) with SRT1720 at $20 \mathrm{mg} / \mathrm{kg}$ immediately following exploratory laparotomy and then once daily for subsequent 2 days. The selected dose was based on previous study [19]. Vehicle-treated mice received 5\%DMSO in saline solution.

\section{Western blot}

Hippocampal tissues were dissected on ice. Then dissected tissues were lysed on ice for 30min in RIPA lysis buffer (Beyotime, Jiangsu, China) with protease and phosphatase inhibitors (Roche, Manheim, Germany). Protein concentration was quantified by BCA protein assay (Thermo Fisher Scientific, Waltham, MA, USA). Protein samples were run on SDS-PAGE gels and then transferred to polyvinylidene fluoride membranes (Millipore, Billerica, MA, USA). Membranes were blocked with $5 \%$ skim milk (Cell Signaling Technology, Beverly, MA, USA) for $1 \mathrm{~h}$ at room temperature, and subsequently incubated with mouse anti-SIRT1 (\#8469, 1:1000, Cell Signaling Technology, Beverly, MA, USA) at $4^{\circ} \mathrm{C}$ overnight. Appropriate DyLight fluorescent dyes secondary antibodies were used (EarthOx, San Francisco, CA, USA). ChemiDoc MP Imaging System (Bio-Rad, Hercules, CA, USA) was used to detect and quantified target bands.

\section{Immunohistochemistry}

Mice were anesthetized and perfused with saline solution and $4 \%$ paraformaldehyde (PFA) sequentially. Harvested brains were post-fixed in $4 \%$ PFA for $24 \mathrm{~h}$, and then cryoprotected in $30 \%$ sucrose for $48 \mathrm{~h}$ at $4^{\circ} \mathrm{C}$. Coronal brain sections (30- $\mu \mathrm{M}$ thickness) were cut using a cryostat microtome. Brain sections were blocked with $5 \%$ bovine serum albumin for $1 \mathrm{~h}$ at room temperature, then incubated with goat anti-ionized calcium binding adapter molecule 1 (Iba1) (\#ab5076, 1:200, abcam, Cambridge, MA, USA), rabbit anti-glia fibrillary acidic protein (GFAP) (\#ab7260, 1:1000, abcam, Cambridge, MA, USA), rabbit anti-Synaptophysin 
(\#A6344, 1:100, abclonal, Wuhan, China), and rabbit anti-lysosomal associated membrane protein 1 (LAMP1) (\#ab24170, 1:200, abcam, Cambridge, MA, USA) at $4^{\circ} \mathrm{C}$ overnight. After washing, brain sections were incubated with fluorophore-conjugated secondary antibodies (1:500, abcam, Cambridge, MA, USA) for $1 \mathrm{~h}$ at room temperature. Laser power and gain were consistent across different experiments. Images were visualized using Leica TCS SP8 STED 3X confocal microscope (Leica, Wetzlar, Germany). Quantitative analyses were performed using Fiji software.

\section{Three-dimensional reconstruction of microglia and engulfment analysis}

Brain sections were imaged with the 100x oil immersion objective /NA1.4 using Leica TCS SP8 STED 3X confocal microscope (Leica, Wetzlar, Germany) with the $0.2 \mathrm{~mm}$ step. Images were acquired under identical settings across all experimental groups. Deconvolution was performed using Huygens Professional software (SVI, Scientific Volume Imaging, Hilversum, the Netherlands). To quantify microglial engulfment of synaptophysin, images were processed using Imaris software (version 9.5.0, Bitplane, Switzerland) based on the protocol from Schafer et al [20]. Microglia and synaptophysin 3D surface rendering were created separately with a threshold. Synaptophysin volume embedded in Iba 1+ structures were considered to be engulfed by microglia.

\section{Golgi staining}

Dendrites of hippocampus were visualized using Golgi-Cox staining kit (\#HTKNS1125, Hitobiotec, Kingsport, TN, USA). Mouse brain were harvested and immersed in impregnation solution for 2 weeks at room temperature, and then transferred to solution for 3 days at $4^{\circ} \mathrm{C}$. Brains were sectioned at a thickness of $150 \mu \mathrm{M}$ using a cryostat microtome, and mounted on gelatinized slides. The slides were further stained in accordance with the manufacturer's instructions. Images were observed with the $100 \mathrm{x}$ oil immersion objective /NA1.4 using Leica TCS SP8 STED 3X confocal microscope (Leica, Wetzlar, Germany). Secondary and third dendrites were sampled for spine density quantification. The counting was conducted by two experimenters independently.

\section{Morris water maze}

Morris water maze has been widely used for assessing spatial learning and memory depending on hippocampal lesions [21]. A round (diameter: $120 \mathrm{~cm}$ ) was filled with $30 \mathrm{~cm}$ depth water at $23 \pm 2^{\circ} \mathrm{C}$. The pool was divided into four quadrants. And the platform was placed $1 \mathrm{~cm}$ underwater at one of quadrants, which was considered as the target quadrants. Non-toxic titanium dioxide powder was premixed into water ensuring platform invisible. Mice were allowed to swim freely for 60 s without the platform to promote familiarity with the testing condition. In the training trials, mice were placed in the pool facing towards the wall at one of releasing points. Mice were given 60 s to find the hidden platform. If not, they were guided to the platform. When mice reached the platform, they were allowed to stay there for $10 \mathrm{~s}$. Training trials were repeated four times per day for 5 consecutive days before surgery, and the releasing points was different for each trial. The probe trial was conducted on the first, third and seventh days after surgery. Mice were released at the opposite quadrant of the hidden platform and swam for 60 s without 
the platform. The escape latency, percentage of time spent in the target quadrant and crossing platform times were analyzed using video-tracking software (ZS Dichuang Techology, Beijing, China).

\section{Y maze}

Spontaneous alternation behavior in $\mathrm{Y}$ maze was recorded to assess working memory [22]. The apparatus consists of three arms with an angel of 120 to each other. Mice were placed at the end of one arm and explored arms freely for $5 \mathrm{~min}$. Arms were labeled $\mathrm{A}, \mathrm{B}$ and $\mathrm{C}$, the sequence of arm entries were recorded. Successful alternation was defined as the exploration of three arms in sequence. The percent alternation was calculated based on the following equation: \%Alternation = [Number of successful alternations/ (Total arm entries-2)] × 100 [23].

\section{Statistical analysis}

Statistical analyses were performed with GraphPad Prism (version 7). Comparisons between two groups were applied using Unpaired Student's $t$-test. One-way analysis of variance (ANOVA) was used for multiple comparisons. Statistical significance was set at $p<0.05$.

\section{Results}

\section{Anesthesia andsurgery resultschanges in gene expression in mice hippocampus}

To investigate the landscape of gene expression after anesthesia and surgery, we performed RNA sequencing in hippocampus from control and surgery group at 72 hours after exploratory laparotomy. Total 29037 genes were identified, among which 844 genes were significant downregulated and 714 genes were significant up-regulated in POCD compared to control group (Fig. 2a). Further, we performed Gene ontology (GO) analysis for significant downregulated genes. Interestingly, the top 22 significant GO terms were enriched in four major aspects of biological processes: (i) calcium ion homeostasis and cell communication; (ii) synapse and dendritic dynamic; (iii) response to cytokines; (iv) learning and memory (Fig. 2b). We next conducted a network analysis of different gene ontology, and found SIRT1, an important deacetylase, not only had a high network score with the most significant GO term (i.e. response to transforming growth factor beta), but also was one of key hubs connecting several significant biological processes (Fig. 2c). These results place SIRT1 as a crucial regulator of biological processes in response to anesthesia and surgery.

\section{Anesthesia and surgery induces SIRT1 downregulation and microglia activation in hippocampus}

To investigate the expression of SIRT1 in hippocampus, we examined protein levels of SIRT1 at $6 \mathrm{~h}, 24 \mathrm{~h}$, and $72 \mathrm{~h}$ after anesthesia and surgery. SIRT1 expression were significantly declined at $24 \mathrm{~h}$ and $72 \mathrm{~h}$ postoperatively $(p<0.01$; Fig. 3a, b). To elucidate the modulatory mechanisms of SIRT1 in POCD, we then identified cellular targets of SIRT1 by immunohistochemistry. We found that SIRT1 co-localized with microglia marker Iba1, but not astrocyte marker GFAP (Fig. 3c). 
Microglia are competent to respond to SIRT1 signaling [24]. We next sought to investigate the impact of anesthesia and surgery on microglia in the hippocampus. Iba1 immunohistochemistry revealed that anesthesia and surgery induced a significant expansion of hippocampal microglia marker in CA1 region (control vs surgery: $p<0.0001$; Fig. 3d, e). Gene set enrichment analyses (GSEA) of our RNA sequencing also showed biological processes of phagosome acidification and phagosome maturation were upregulated in surgery group when compared with control group (Fig. 3f, g).

\section{SIRT1 activation prevents alteration in microglia morphology after anesthesia and surgery}

Microglia activation is correlated with specific morphologic alteration [25]. To investigate whether SIRT1 activation could affect microglia activation after anesthesia and surgery, we administrated SRT1720, an agonist of SIRT1, intraperitoneally in mice of surgery group. Quantitative morphometric 3D measurements of microglia found no significant difference in surface area $(p=0.8811$; Fig. $4 \mathrm{a}, \mathrm{b})$ and microglia size ( $p=0.3919$; Fig. $4 a, c)$ among three groups. We further calculated soma size and occupied area of microglia. Compared with control, anesthesia and surgery increased soma size of microglia in hippocampus (control vs surgery-vehicle, $p<0.0001$; Fig. $4 \mathrm{~d}$, e), and decreased the occupied area (control vs surgery-vehicle, $p<0.0001 ;$ Fig. $4 \mathrm{f}, \mathrm{g}$ ). Notably, alteration in microglia morphology after anesthesia and surgery was reversed by SIRT1 agonist, with decreased soma size (surgery-vehicle vs surgery-SRT1720, $p$ $<0.0001$; Fig. 4d, e) and increased occupied area (surgery-vehicle vs surgery-SRT1720, $p<0.0001$; Fig. 4f, g).

\section{SIRT1 activation ameliorates microglia mediated synaptic engulfment after anesthesia and surgery}

Microglia are implicated in synaptic engulfment, which contributes to cognitive impairment [26]. Interestingly, genes related with synaptic protein were downregulated in our surgery group (Fig. 5a). Thus, we evaluated synapse engulfment by measuring presynaptic marker synaptophysin in microglia. 3D surface reconstruction and rendering showed that the volume of synaptophysin within microglia were significantly increased in mice after anesthesia and surgery (control vs surgery-vehicle, $p<0.0001$; Fig. $5 b, c)$, which were ameliorated by SIRT1 activation (surgery-vehicle vs surgery-SRT1720, $p<0.0001$; Fig. $5 b, c)$.

We next detected lysosomal marker LAMP1 enriched in dystrophic neurites. There was a significant elevation of LAMP1 in dentate gyrus (DG) of hippocampus in surgery group (control vs surgery-vehicle, $p$ $<0.0001$; Fig. 5d, e). Surgery induced LAMP1 elevation were reversed by SIRT1 activation (surgery-vehicle vs surgery-SRT1720, $p<0.0001$; Fig. $5 d$, e).

\section{SIRT1 activation restores anesthesia and surgery-induced spine loss}

To further assess the impact of microglia engulfment on synaptic structures, we performed Golgi staining on hippocampus. We found that surgery induced spine loss of neurons in CA1 region (control vs surgeryvehicle, $p<0.0001$; Figure 6a, b, d), which were alleviated were rescued by SRT1720 treatment (surgeryvehicle vs surgery-SRT1720, $p<0.0001$; Figure $6 a, b, d)$. We also found reduced spine density in neurons 
of DG (control vs surgery-vehicle, $p<0.05$; Figure $6 \mathrm{~b}, \mathrm{c}$ ) and CA3 region (control vs surgery-vehicle, $p<$ 0.0001 ; Figure $6 \mathrm{~b}, \mathrm{e}$ ) after anesthesia and surgery. Besides, SIRT1 activation partially restore the spine loss (surgery-vehicle vs surgery-SRT1720, $p<0.0001$; Fig. 6b, c, e).

\section{SIRT1 activation alleviate cognitive deficit after anesthesia and surgery}

To further study the effects of SIRT1 on cognitive performance, tests of $Y$ maze were performed to evaluate working memory. We found similar performance on percentage of spontaneous alteration among groups ( $p=0.9343 ; \mathrm{Fig} .7 \mathrm{a})$. In addition, we assess reference spatial memory using Morris water maze. Escape latency decreased across training days ( $p<0.0001$; Fig. 7b), and no significant difference among groups ( $p=0.9988 ;$ Fig. 7b). Probe trials were conducted postoperative day (POD) 1 and 3 . Compare to control group, a statistically significant reduction in percentage of time spent in target quadrant was observed in mice after anesthesia and surgery on POD1 (control vs surgery-vehicle, $p=$ 0.0044; Fig. 7c). Mice in surgery-vehicle group also displayed a fewer crossing times of the platform on POD1 (control vs surgery-vehicle, $p=0.0014$ ) and POD3 (control vs surgery-vehicle, $p=0.0278 ;$ Fig. $7 \mathrm{~d}$ ). We found that SRT1720 treated group exhibited improve percentage of time spent in target quadrant on POD1 and POD3 (surgery-vehicle vs surgery-SRT1720, $p<0.05$; Fig. 7c)

\section{Discussion}

In this study, we found that anesthesia and surgery induced SIRT1 downregulation in hippocampus. Microglia abnormal activation after anesthesia and surgery led to synaptic engulfment and spine loss, resulting in cognitive impairment, all of which were alleviated by SRT1720 treatment. Thus, our data suggest the important roles of increasing SIRT1 function on protection against postoperative cognitive dysfunction.

Postoperative cognitive dysfunction is a common neurological complication for surgical patients, especially for the elderly [27]. Microglia activation and neuroinflammation are long thought as the underlying mechanisms of POCD [2]. Microglia serve as the innate immune cells in brain, surveilling and maintaining the central nervous system homeostasis [8]. Proliferation and activation of microglia is a key feature in neurodegenerative disease settings [28]. Our data showing microglia activation following anesthesia and surgery are in line with several studies [29-31]. Leakage of peripheral cytokines through blood-brain barrier breakdown might be the main trigger [2]. Work in animal models of other neurodegenerative or neurological disease have shown alteration in microglia morphology during activation [32,33]. Ramified microglia convert to amoeboid subtype, which is characterized by expanded cell bodies and retracted branches [34]. Although no notable changes were observed in surface area and microglia size, we found decreased occupied area and increased soma size of microglia in hippocampus after anesthesia and surgery.

A strong correlation has been demonstrated between morphologic alteration and microglia functional plasticity [35]. Exacerbated phagocytic activity could shape dendrites and engulf synaptic terminals, which then leads to cognitive impairments in Alzheimer's disease [28], epilepsy [36] and demyelinating 
disease [33]. RNA sequencing and GSEA of surgery versus control mice in our study revealed progressive increases in phagosome acidification and phagosome maturation genes, as well as downregulation of synaptic protein genes. We previously demonstrated that loss of synaptic protein correlated with microglial phagocytosis [12]. Using 3D reconstruction in current study, we directly observed microglia in mice after anesthesia and surgery contained a higher amount of engulfed synaptophysin than those in control group. In concert with microglial phagocytosis, the lysosome marker LAMP1, which is enriched in dystrophic neurites, was also accumulated after anesthesia and surgery. Together, our data enforce the picture that microglia mediated synaptic engulfment may be a key question of POCD.

As a consequence of excessive synaptic engulfment, reduction of dendritic spines is pathogenic in schizophrenia [37], associated with forgetting of memory [26]. In our study, anesthesia and surgery significantly reduced the spine density in various area of hippocampus, as detected by Golgi staining. Our data are consistent with previous work showing dendritic spine loss in POCD [38]. In our study, anesthesia and surgery did not lead to deficits in working memory, but it produced long-term spatial memory impairment. In agreement with the key role of hippocampus for long-term memory.

In our study, Gene Ontology and network analysis indicated that SIRT1 were downregulated upon anesthesia and surgery, and was a hub gene of enriched pathways. SIRT1 is one of (NAD+)-dependent deacetylases that modulate multiple cellular processes [14]. SIRT1 was reported to be protective in different neurodegenerative diseases [16] and psychiatric disorders [15]. We observed a decreased SIRT1 expression in the hippocampus 1 and 3 days after anesthesia and surgery. Our results are in keeping with recent works $[39,40]$. SIRT1 is enriched in the nuclei and cytoplasm of microglia, implicating microglia as the target of SIRT1 in hippocampus. Here, we found SIRT1 activation dampens alteration in microglia morphology induced by anesthesia and surgery. The ameliorative microglia engulfment of synaptic protein detected in POCD mice with additional SRT1720 administration is paralleled by the restored spine loss. In line with changes determined in the hippocampus, SRT1720 administration was beneficial for behavioral defects in mice after anesthesia and surgery. Consistent with this protective effects, SRT1720 significantly reduced plasma inflammatory cytokine levels in mice after cardiac surgery [41], which can trigger microglia activation.

There are some limitations in our study. The sample size for RNA sequencing is small. Besides, we did not demonstrate the engulfment on postsynaptic elements. We did not demonstrate the signaling mechanisms by which SIRT1 overexpression prevents microglia activation. Previous study revealed a potential link between SIRT1 activation and inhibition of nuclear factor-kappa B (NF-kB) in vitro [42]. To addressing these issues, further investigation is needed.

\section{Conclusions}

In summary, abnormal microglia activation mediates synaptic engulfment could be responsible for postoperative cognitive dysfunction. SIRT1 activation after anesthesia and surgery alleviates microglia 
activation, inhibits the engulfment of synapse elements, reduces spine loss and improves cognitive performance. Increasing SIRT1 function may be a promising therapeutic strategy for POCD.

\section{Abbreviations}

POCD: Postoperative cognitive dysfunction; SIRT1: Silent information regulator 1; NAD: Nicotinamide adenine dinucleotide; NF-kB: Nuclear factor-kappa B; FOXO: Forkhead box O; PPARa: Peroxisome proliferator-activated receptor alpha; PGC-1a: PPAR-gamma co-activator 1-alpha; CNS: Central nervous system; DMSO: Dimethyl sulfoxide; BCA: Bicinchoninic acid; PFA: Paraformaldehyde; Iba1: Ionized calcium binding adapter molecule 1; GFAP: Glia fibrillary acidic protein; SYP: Synaptophysin; LAMP1: Lysosomal associated membrane protein 1; MWM: Morris water maze; ANOVA: One-way analysis of variance; POD: Postoperative day

\section{Declarations}

\section{Funding}

This study was supported by the Beijing Hospitals Authority Youth Programme (QML20190307), The Youth Backbone of Beijing Outstanding Talent Training Project (2018000021469G215), Beijing Natural Science Foundation (7194270), National Natural Science Foundation of China (81771139 and 82071176).

\section{Competing interests}

The authors declare that they have no competing interests.

\section{Availability of data and materials}

The datasets used and/or analyzed in the present study are available from the corresponding author upon reasonable request.

\section{Authors' contributions}

Designed Research, C.W. and Y.S.; Performed Research, Y.S., F.Y. and D.L.; Data Analyzed, Y.S. and Y.W.; Writing-Original Draft Preparation, Y.S.; Writing-Review \& Editing, C.W., Y.W. and H.S.; Supervision, Y.Z. and A.W.; Funding Acquisition, C.W. and A.W. The final manuscript was approved by all authors.

\section{Ethics approval and consent to participate}

All procedures were approved by Institutional Animal Care and Use Committee at Capital Medical University (protocol AEEI-2020-117)

Consent for publication 
Not applicable.

\section{Acknowledgements}

We thank the assistance of the National Center for Protein Sciences at Peking University in Beijing, China, and the State Key Laboratory of Membrane Biology at Peking University in Beijing, China.

\section{References}

1. Evered L, Silbert B, Knopman DS, Scott DA, DeKosky ST, Rasmussen LS, Oh ES, Crosby G, Berger M, Eckenhoff RG, Nomenclature Consensus Working G (2018) Recommendations for the Nomenclature of Cognitive Change Associated with Anaesthesia and Surgery-2018. Anesthesiology 129 (5):872879. doi:10.1097/ALN.0000000000002334

2. Skvarc DR, Berk M, Byrne LK, Dean OM, Dodd S, Lewis M, Marriott A, Moore EM, Morris G, Page RS, Gray L (2018) Post-Operative Cognitive Dysfunction: An exploration of the inflammatory hypothesis and novel therapies. Neurosci Biobehav Rev 84:116-133. doi:10.1016/j.neubiorev.2017.11.011

3. Rosczyk HA, Sparkman NL, Johnson RW (2008) Neuroinflammation and cognitive function in aged mice following minor surgery. Exp Gerontol 43 (9):840-846. doi:10.1016/j.exger.2008.06.004

4. Li Z, Liu F, Ma H, White PF, Yumul R, Jiang Y, Wang N, Cao X (2017) Age exacerbates surgery-induced cognitive impairment and neuroinflammation in Sprague-Dawley rats: the role of IL-4. Brain Res 1665:65-73. doi:10.1016/j.brainres.2017.04.004

5. Wang HL, Liu H, Xue ZG, Liao QW, Fang H (2016) Minocycline attenuates post-operative cognitive impairment in aged mice by inhibiting microglia activation. J Cell Mol Med 20 (9):1632-1639. doi:10.1111/jcmm.12854

6. Vizcaychipi MP, Watts HR, O'Dea KP, Lloyd DG, Penn JW, Wan Y, Pac-Soo C, Takata M, Ma D (2014) The therapeutic potential of atorvastatin in a mouse model of postoperative cognitive decline. Ann Surg 259 (6):1235-1244. doi:10.1097/SLA.0000000000000257

7. Swinnen N, Smolders S, Avila A, Notelaers K, Paesen R, Ameloot M, Brone B, Legendre P, Rigo JM (2013) Complex invasion pattern of the cerebral cortex bymicroglial cells during development of the mouse embryo. Glia 61 (2):150-163. doi:10.1002/glia.22421

8. Marquez-Ropero M, Benito E, Plaza-Zabala A, Sierra A (2020) Microglial Corpse Clearance: Lessons From Macrophages. Front Immunol 11:506. doi:10.3389/fimmu.2020.00506

9. Paolicelli RC, Bolasco G, Pagani F, Maggi L, Scianni M, Panzanelli P, Giustetto M, Ferreira TA, Guiducci E, Dumas L, Ragozzino D, Gross CT (2011) Synaptic pruning by microglia is necessary for normal brain development. Science 333 (6048):1456-1458. doi:10.1126/science.1202529

10. Salter MW, Stevens B (2017) Microglia emerge as central players in brain disease. Nat Med 23 (9):1018-1027. doi:10.1038/nm.4397

11. Cardozo PL, de Lima IBQ, Maciel EMA, Silva NC, Dobransky T, Ribeiro FM (2019) Synaptic Elimination in Neurological Disorders. Curr Neuropharmacol 17 (11):1071-1095. 
doi:10.2174/1570159X17666190603170511

12. Xiong C, Liu J, Lin D, Zhang J, Terrando N, Wu A (2018) Complement activation contributes to perioperative neurocognitive disorders in mice. J Neuroinflammation 15 (1):254. doi:10.1186/s12974-018-1292-4

13. Fujita Y, Yamashita T (2018) Sirtuins in Neuroendocrine Regulation and Neurological Diseases. Front Neurosci 12:778. doi:10.3389/fnins.2018.00778

14. Donmez G, Outeiro TF (2013) SIRT1 and SIRT2: emerging targets in neurodegeneration. EMBO Mol Med 5 (3):344-352. doi:10.1002/emmm.201302451

15. Abe-Higuchi N, Uchida S, Yamagata H, Higuchi F, Hobara T, Hara K, Kobayashi A, Watanabe Y (2016) Hippocampal Sirtuin 1 Signaling Mediates Depression-like Behavior. Biol Psychiatry 80 (11):815-826. doi:10.1016/j.biopsych.2016.01.009

16. Gan BQ, Tang BL (2010) Sirt1's beneficial roles in neurodegenerative diseases - a chaperonin containing TCP-1 (CCT) connection? Aging Cell 9 (5):924-929. doi:10.1111/j.14749726.2010.00597.x

17. Pan K, Li X, Chen Y, Zhu D, Li Y, Tao G, Zuo Z (2016) Deferoxamine pre-treatment protects against postoperative cognitive dysfunction of aged rats by depressing microglial activation via ameliorating iron accumulation in hippocampus. Neuropharmacology 111:180-194. doi:10.1016/j.neuropharm.2016.09.004

18. Liu L, Si L, Meng X, Luo L (2015) Comparative transcriptomic analysis reveals novel genes and regulatory mechanisms of Tetragenococcus halophilus in response to salt stress. $\mathrm{J}$ Ind Microbiol Biotechnol 42 (4):601-616. doi:10.1007/s10295-014-1579-0

19. Li L, Tan HP, Liu CY, Yu LT, Wei DN, Zhang ZC, Lu K, Zhao KS, Maegele M, Cai DZ, Gu ZT (2019) Polydatin prevents the induction of secondary brain injury after traumatic brain injury by protecting neuronal mitochondria. Neural Regen Res 14 (9):1573-1582. doi:10.4103/1673-5374.255972

20. Schafer DP, Lehrman EK, Heller CT, Stevens B (2014) An engulfment assay: a protocol to assess interactions between CNS phagocytes and neurons. J Vis Exp (88). doi:10.3791/51482

21. Vorhees CV, Williams MT (2006) Morris water maze: procedures for assessing spatial and related forms of learning and memory. Nat Protoc 1 (2):848-858. doi:10.1038/nprot.2006.116

22. Lalonde R (2002) The neurobiological basis of spontaneous alternation. Neurosci Biobehav Rev 26 (1):91-104. doi:10.1016/s0149-7634(01)00041-0

23. Rosenzweig N, Dvir-Szternfeld R, Tsitsou-Kampeli A, Keren-Shaul H, Ben-Yehuda H, Weill-Raynal P, Cahalon L, Kertser A, Baruch K, Amit I, Weiner A, Schwartz M (2019) PD-1/PD-L1 checkpoint blockade harnesses monocyte-derived macrophages to combat cognitive impairment in a tauopathy mouse model. Nat Commun 10 (1):465. doi:10.1038/s41467-019-08352-5

24. Merlo S, Luaces JP, Spampinato SF, Toro-Urrego N, Caruso GI, D'Amico F, Capani F, Sortino MA (2020) SIRT1 Mediates Melatonin's Effects on Microglial Activation in Hypoxia: In Vitro and In Vivo Evidence. Biomolecules 10 (3). doi:10.3390/biom10030364 
25. Torres-Platas SG, Comeau S, Rachalski A, Bo GD, Cruceanu C, Turecki G, Giros B, Mechawar N (2014) Morphometric characterization of microglial phenotypes in human cerebral cortex. $\mathrm{J}$ Neuroinflammation 11:12. doi:10.1186/1742-2094-11-12

26. Wang C, Yue H, Hu Z, Shen Y, Ma J, Li J, Wang XD, Wang L, Sun B, Shi P, Wang L, Gu Y (2020) Microglia mediate forgetting via complement-dependent synaptic elimination. Science 367 (6478):688-694. doi:10.1126/science.aaz2288

27. Evered L, Scott DA, Silbert B, Maruff $P$ (2011) Postoperative cognitive dysfunction is independent of type of surgery and anesthetic. Anesth Analg 112 (5):1179-1185.

doi:10.1213/ANE.0b013e318215217e

28. Hansen DV, Hanson JE, Sheng M (2018) Microglia in Alzheimer's disease. J Cell Biol 217 (2):459-472. doi: $10.1083 /$ jcb. 201709069

29. Hovens IB, Schoemaker RG, van der Zee EA, Heineman E, Nyakas C, van Leeuwen BL (2013) Surgeryinduced behavioral changes in aged rats. Exp Gerontol 48 (11):1204-1211. doi:10.1016/j.exger.2013.07.011

30. Wang Y, Cao X, Ma H, Tan W, Zhang L, Li Z, Gao Y (2016) Prior stressor exposure delays the recovery of surgery-induced cognitive impairment and prolongs neuroinflammation in aged rats. Brain Res 1648 (Pt A):380-386. doi:10.1016/j.brainres.2016.07.045

31. Zhang Q, Zhang J, Yan Y, Zhang P, Zhang W, Xia R (2017) Proinflammatory cytokines correlate with early exercise attenuating anxiety-like behavior after cerebral ischemia. Brain Behav 7 (11):e00854. doi:10.1002/brb3.854

32. Xu ZX, Kim GH, Tan JW, Riso AE, Sun Y, Xu EY, Liao GY, Xu H, Lee SH, Do NY, Lee CH, Clipperton-Allen AE, Kwon S, Page DT, Lee KJ, Xu B (2020) Elevated protein synthesis in microglia causes autism-like synaptic and behavioral aberrations. Nat Commun 11 (1):1797. doi:10.1038/s41467-020-15530-3

33. Werneburg S, Jung J, Kunjamma RB, Ha SK, Luciano NJ, Willis CM, Gao G, Biscola NP, Havton LA, Crocker SJ, Popko B, Reich DS, Schafer DP (2020) Targeted Complement Inhibition at Synapses Prevents Microglial Synaptic Engulfment and Synapse Loss in Demyelinating Disease. Immunity 52 (1):167-182 e167. doi:10.1016/j.immuni.2019.12.004

34. Yuan T, Manohar K, Latorre R, Orock A, Greenwood-Van Meerveld B (2020) Inhibition of Microglial Activation in the Amygdala Reverses Stress-Induced Abdominal Pain in the Male Rat. Cell Mol Gastroenterol Hepatol 10 (3):527-543. doi:10.1016/j.jcmgh.2020.04.020

35. Diaz-Aparicio I, Beccari S, Abiega O, Sierra A (2016) Clearing the corpses: regulatory mechanisms, novel tools, and therapeutic potential of harnessing microglial phagocytosis in the diseased brain. Neural Regen Res 11 (10):1533-1539. doi:10.4103/1673-5374.193220

36. Andoh M, Ikegaya Y, Koyama R (2019) Synaptic Pruning by Microglia in Epilepsy. J Clin Med 8 (12). doi: $10.3390 / \mathrm{jcm} 8122170$

37. Sellgren CM, Gracias J, Watmuff B, Biag JD, Thanos JM, Whittredge PB, Fu T, Worringer K, Brown HE, Wang J, Kaykas A, Karmacharya R, Goold CP, Sheridan SD, Perlis RH (2019) Increased synapse 
elimination by microglia in schizophrenia patient-derived models of synaptic pruning. Nat Neurosci 22 (3):374-385. doi:10.1038/s41593-018-0334-7

38. Qiu LL, Pan W, Luo D, Zhang GF, Zhou ZQ, Sun XY, Yang JJ, Ji MH (2020) Dysregulation of $\mathrm{BDNF} / \mathrm{TrkB}$ signaling mediated by NMDAR/Ca(2+)/calpain might contribute to postoperative cognitive dysfunction in aging mice. J Neuroinflammation 17 (1):23. doi:10.1186/s12974-019-1695$\mathrm{x}$

39. Yan J, Luo A, Sun R, Tang X, Zhao Y, Zhang J, Zhou B, Zheng H, Yu H, Li S (2020) Resveratrol Mitigates Hippocampal Tau Acetylation and Cognitive Deficit by Activation SIRT1 in Aged Rats following Anesthesia and Surgery. Oxid Med Cell Longev 2020:4635163. doi:10.1155/2020/4635163

40. Yan J, Luo A, Gao J, Tang X, Zhao Y, Zhou B, Zhou Z, Li S (2019) The role of SIRT1 in neuroinflammation and cognitive dysfunction in aged rats after anesthesia and surgery. Am $\mathrm{J}$ Transl Res 11 (3):1555-1568

41. Shi J, Zou X, Jiang K, Wang F (2019) SIRT1 mediates improvement of cardiac surgery induced postoperative cognitive dysfunction via TLR4/NF-kappaB pathway. World J Biol Psychiatry:1-21. doi:10.1080/15622975.2019.1656820

42. Tong Y, Fu H, Xia C, Song W, Li Y, Zhao J, Zhang X, Gao X, Yong J, Liu Q, Yang C, Wang H (2020) Astragalin Exerted Antidepressant-like Action through SIRT1 Signaling Modulated NLRP3 Inflammasome Deactivation. ACS Chem Neurosci 11 (10):1495-1503. doi:10.1021/acschemneuro.0c00156

\section{Figures}



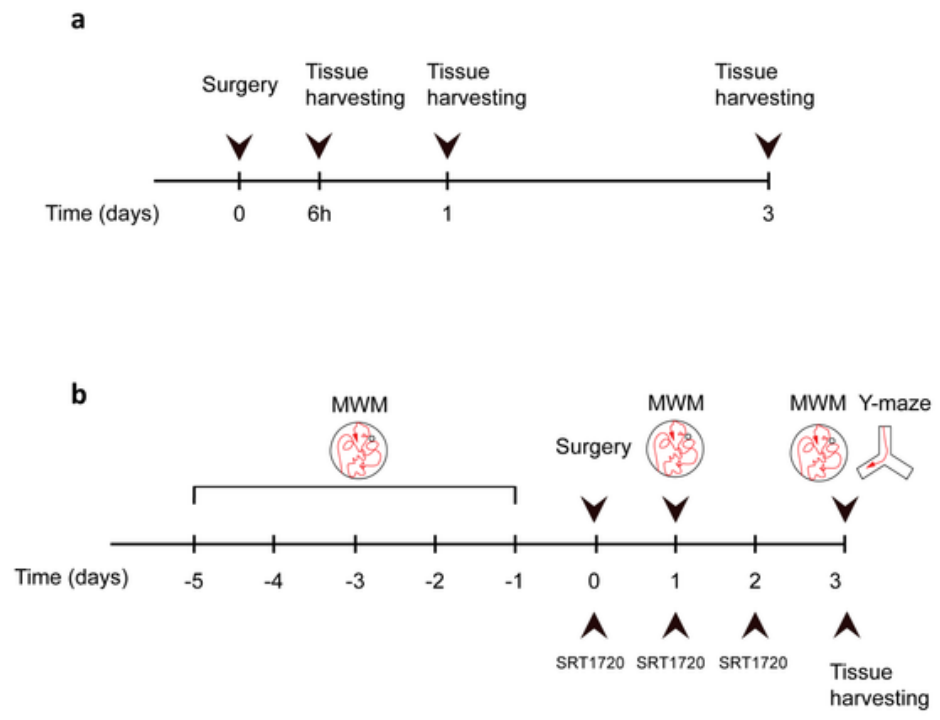

\section{Figure 1}

Schematic of the experimental design. a. Mice were assigned to control and surgery group. Tissue were harvested at $6 \mathrm{~h}, 1$ and 3 days after surgery. b. POCD mice were treated with either SRT1720 or IgG. Tissue were harvested at postoperative day 3. Training trials of Morris water maze were performed 5 consecutive days before surgery. Probe trials were performed postoperative day 1 and 3. Y maze were conducted on postoperative day 3. MWM, Morris water maze. 
b

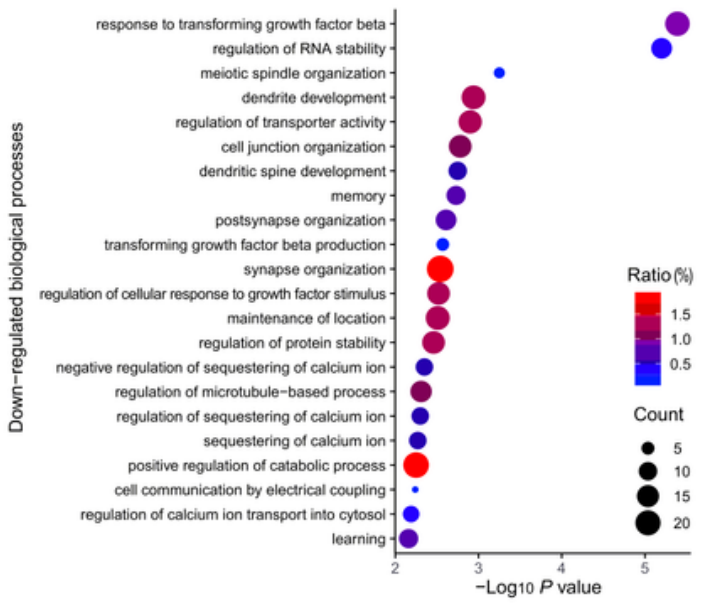

c

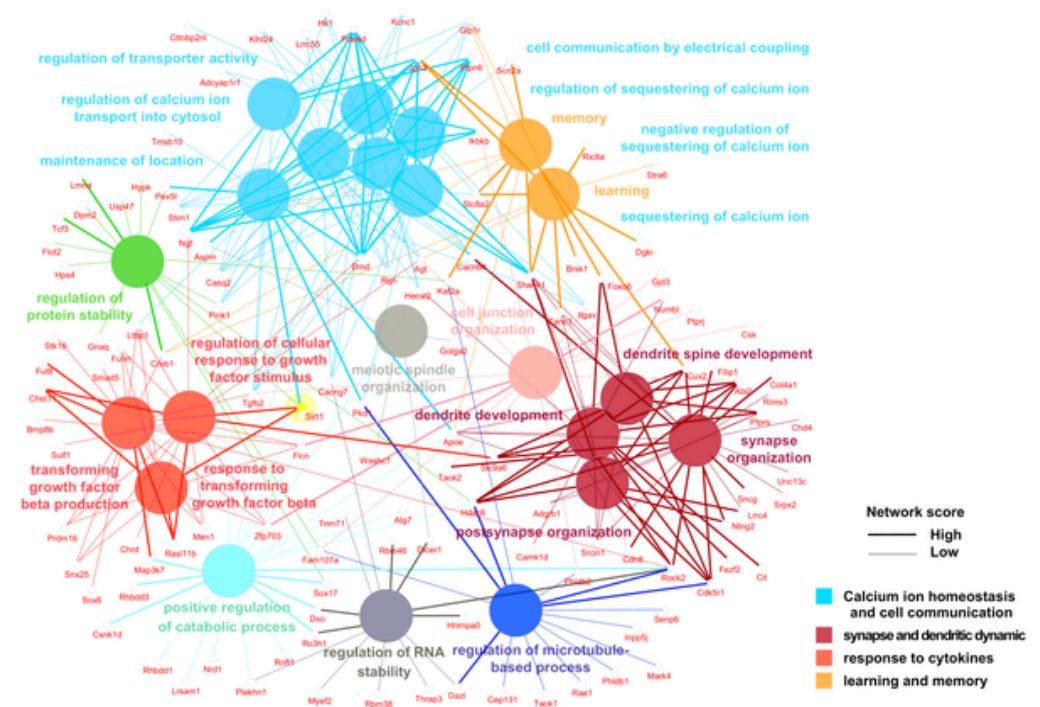

Figure 2

Panorama of RNA changes when compared POCD to control group. a. Volcano plot representation of differentially expressed gene distribution $(p<0.05)$. b. Dot plot of top 22 Gene oncology terms for downregulated biological processes. c. Network analysis of top 22 downregulated biological processes among the gene interaction networks. 
a

b
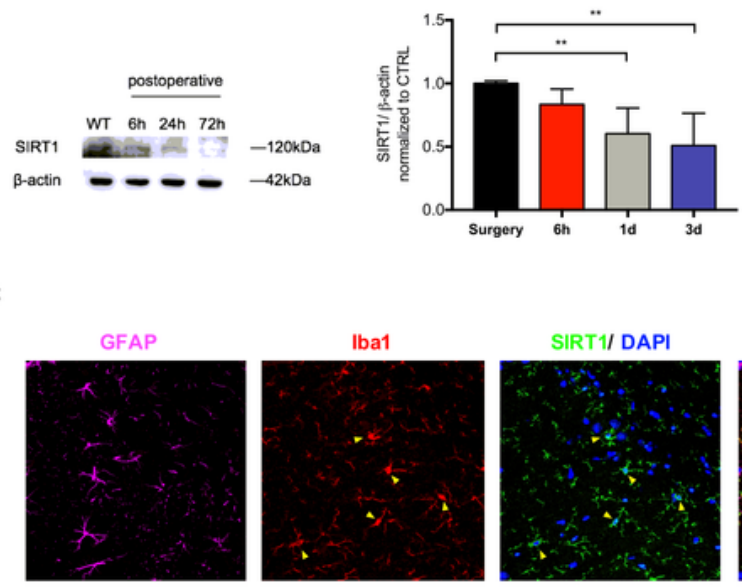

SIRT1/ DAPI
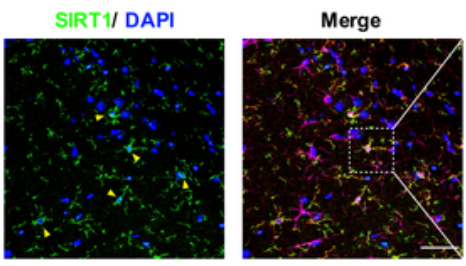

Iba1/ SIRT1/ DAPI

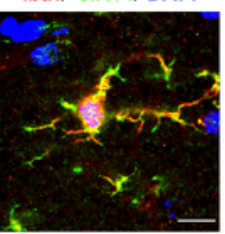

d

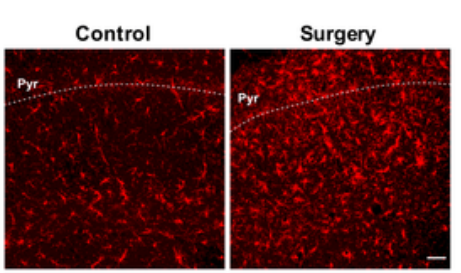

e

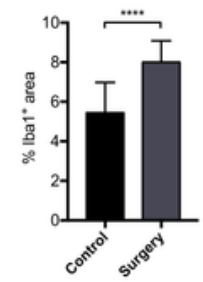

f

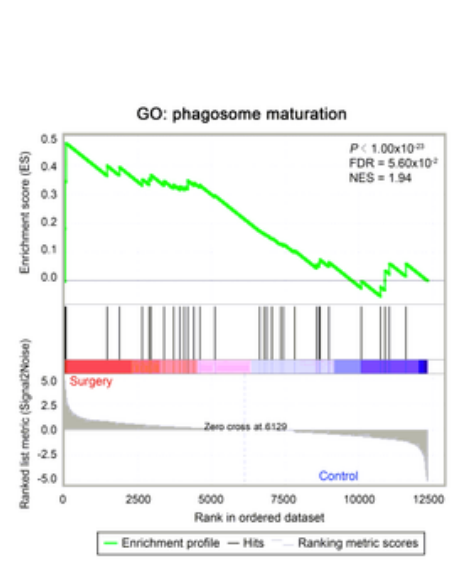

g
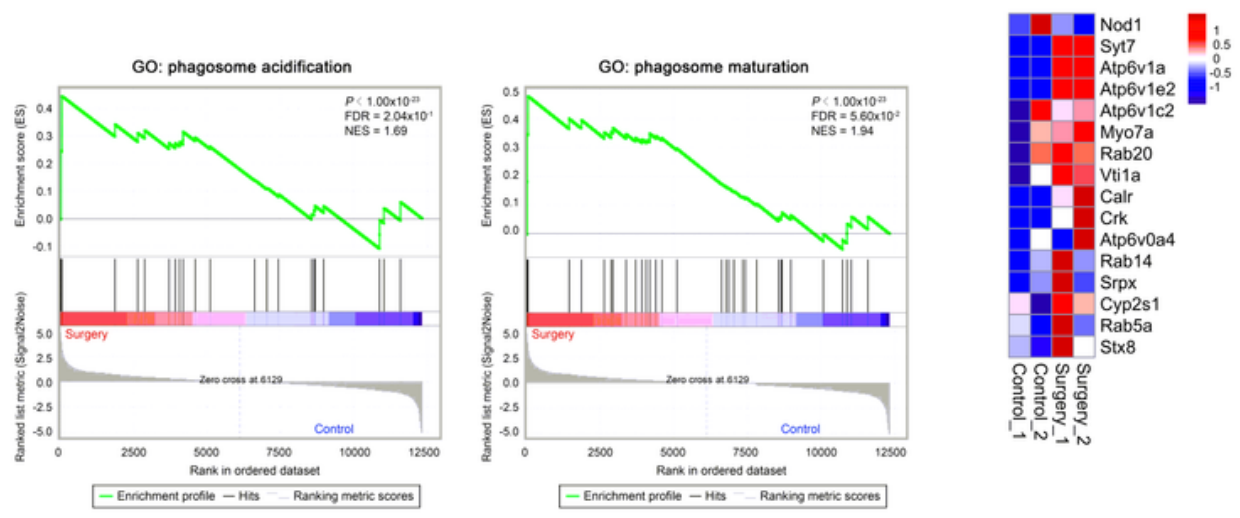

\section{Figure 3}

Anesthesia and surgery induces microglia activation in the hippocampus. a. Time course of SIRT1 expression. One-way ANOVA with Dunnett post-hoc test. b. Qualification of (a). c. Representative images of SIRT1 co-labled with antibodies to cell-type-specific markers, $n=5$. Upper panel scale bar, 50 $\mu \mathrm{m}$; lower

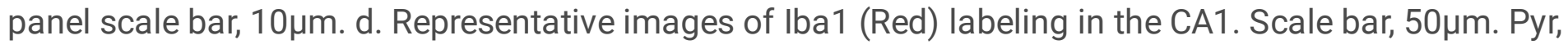
pyramidal cell layer. e. Qualification of (d), $n=5$ per group. Two sided t-test. f. Gene set enrichment 
analyses (GSEA) of phagosome acidification (left) and phagosome maturation (right) in surgery versus control group. NES: normalized enrichment score; FDR: false discovery rate. g. Heatmap representation of differential expression for phagosome acidification and phagosome maturation genes. Data are shown as mean $\pm \mathrm{SD}$. ${ }^{\star *} \mathrm{p}<0.01 ; * \star \star * \mathrm{p}<0.0001$.

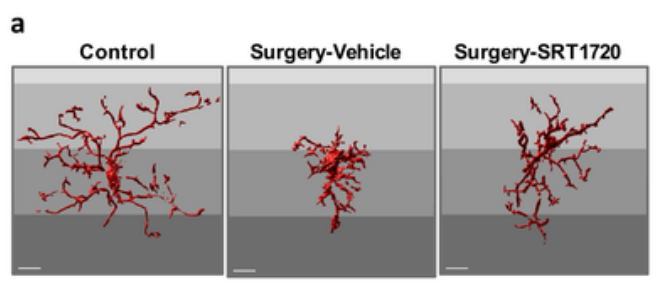

b

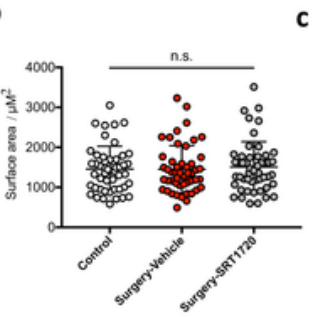

c

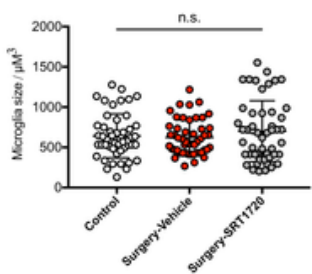

d

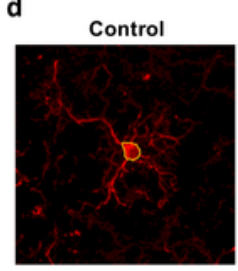

f

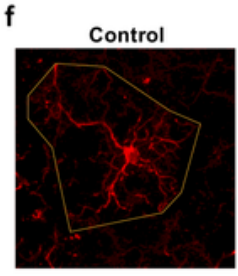

Surgery-Vehicle
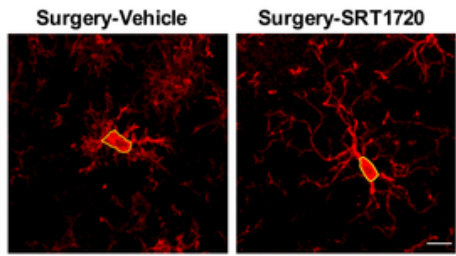

Surgery-Vehicle

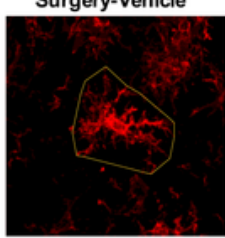

Surgery-SRT1720

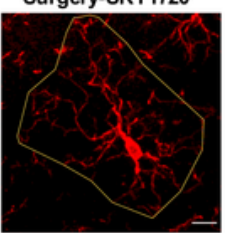

e

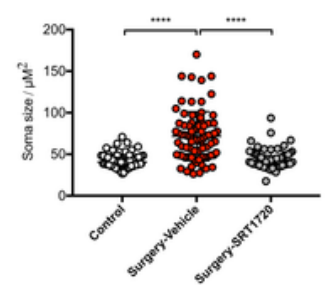

g

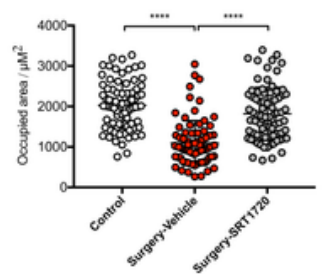

Figure 4 
SIRT1 activation prevents alteration in microglia morphology after anesthesia and surgery. a.

Representative 3D reconstruction and rendering of microglia. Scale bar,10 $\mu \mathrm{m}$. b. Quantification of surface area of microglia, $n=5$ per group; 10 cells per mouse were quantified. c. Quantification of microglia size, $\mathrm{n}=5$ per group; 10 cells per mouse were quantified. $d$. Representative images of microglial (Iba1, red) and showing measurement of soma size (outlined by yellow line). Scale bar, $10 \mu \mathrm{m}$. e. Showing measurement of occupied area (outlined by yellow line). Scale bar, $10 \mu \mathrm{m}$. $\mathrm{f}$. Quantification of (d), $n=5$ per group; 14-18 cells per mouse were quantified. g. Quantification of (e), $n=5$ per group; $14-18$ cells per mouse were quantified. One-way ANOVA with Tukey post-hoc test. All data are shown as mean \pm SD. n.s. not significant; $* \star \star \star p ~<0.0001$. 

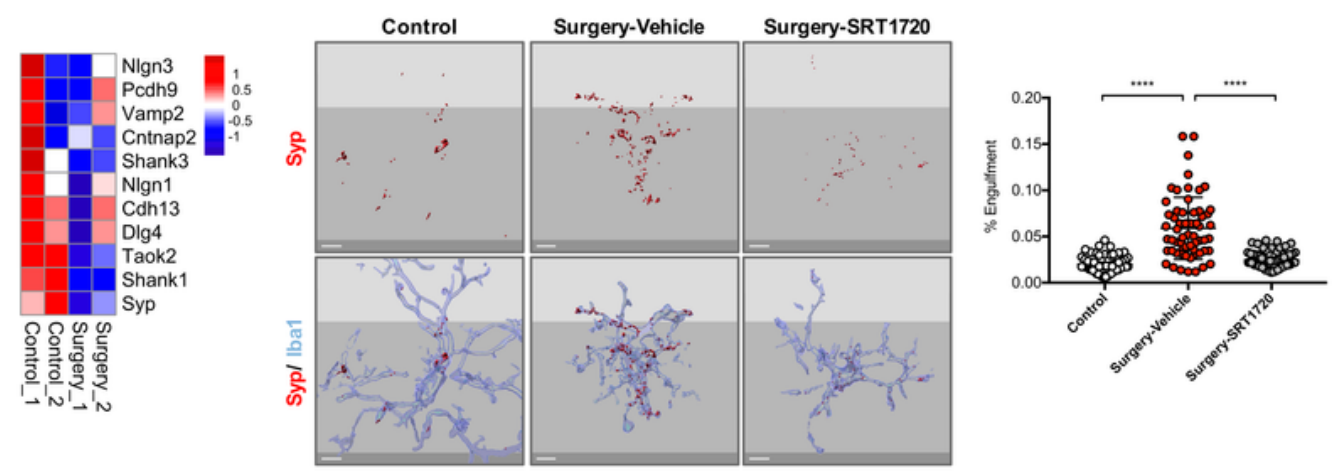

d

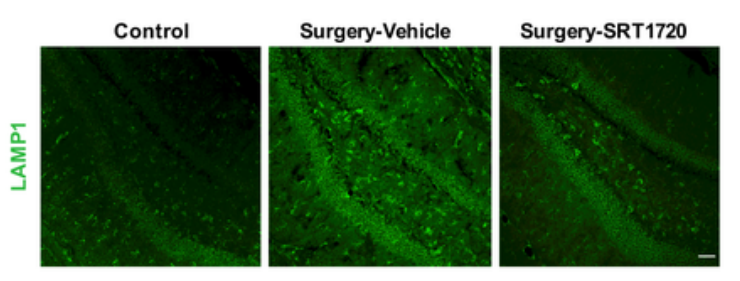

e

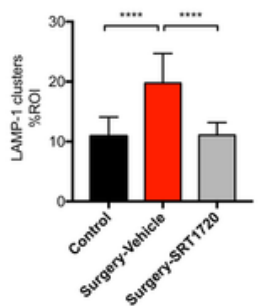

\section{Figure 5}

SIRT1 overexpression ameliorates microglia engulfment of presynaptic inputs after surgery. a. Heatmap of synaptic protein related gene expression. b. Representative 3D reconstruction and rendering of Syp signals inside Iba1+microglia from each experimental group. Scale bar, $5 \mu \mathrm{m}$. c. Quantification of (b), $\mathrm{n}=$ 5 per group; $10-15$ cells per mouse were quantified. d. Maximum z projections showing LAMP1 in the dentate gyrus of hippocampus from each experimental group. Scale bar, $50 \mu \mathrm{m}$. e. Quantification of (d), $n$ 


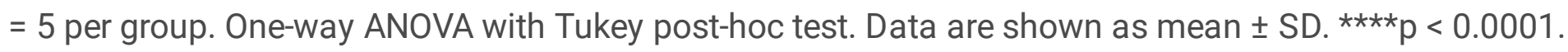
Syp, synaptophysin.

a

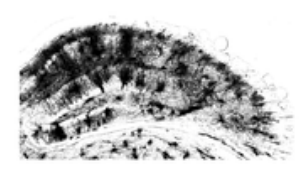

b

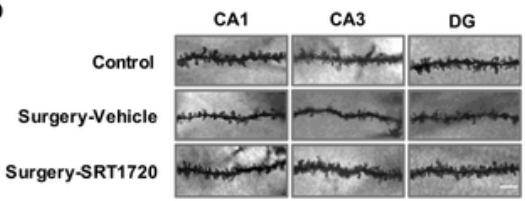

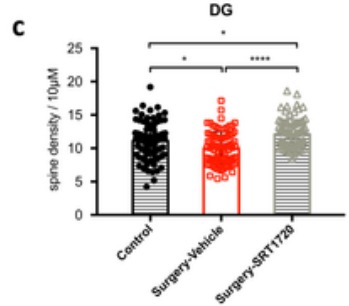

e d

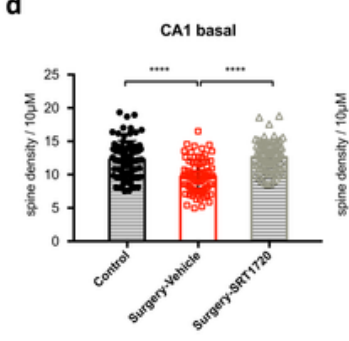

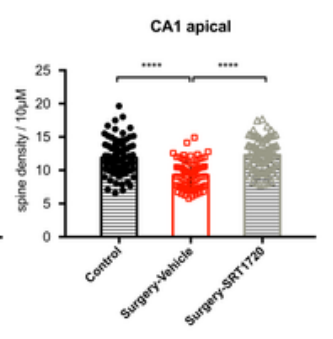
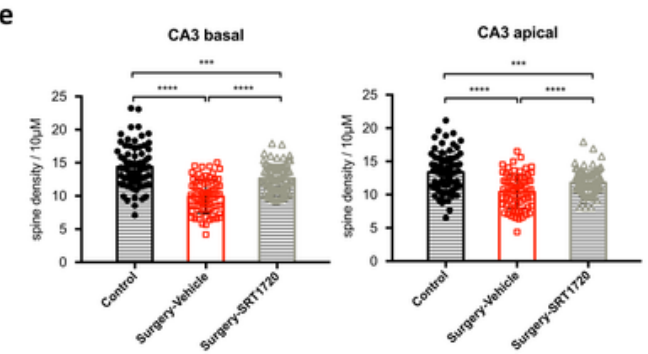

\section{Figure 6}

SIRT1 activation restores surgery-induced spine loss. a. Representative images of Golgi staining of hippocampus. b. High-magnification representative images of dendritic spine from each experimental group. Scale bar, $5 \mu$ m. c. Spine density of DG neurons. $n=80-84$ spines from 4 mice per group. d. Spine 
density in apical and basal dendrites of CA1 neurons, $n=78-80$ spines from 4 mice per group. e. Spine density in apical and basal dendrites of CA3 neurons. $n=74-80$ spines from 4 mice per group. One-way ANOVA with Tukey post-hoc test. All data are shown as mean \pm SD. ${ }^{\star} p<0.05 ;{ }^{*} p<0.01 ;{ }^{* \star *} p<0.001$;

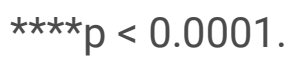

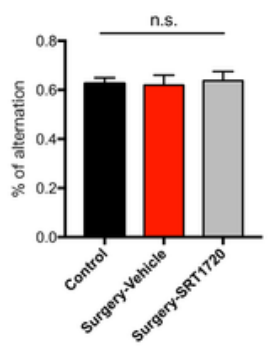

c

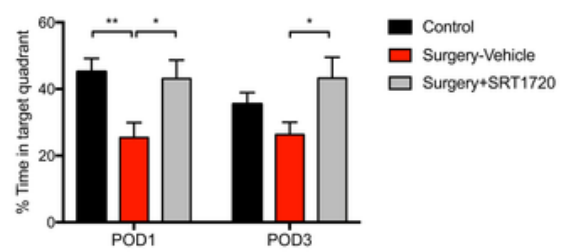

b

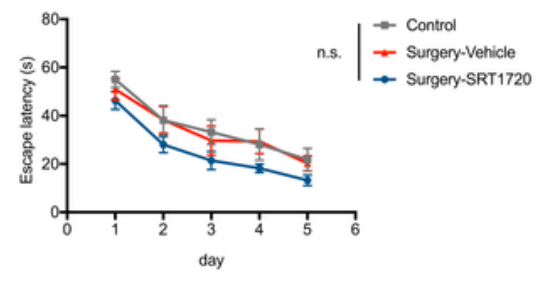

d

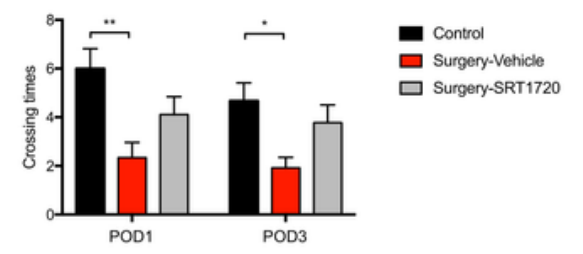

Figure 7 
SIRT1 activation overcomes loss of cognitive performance after surgery and anesthesia. a. Y-maze performance on the third day after surgery. $n=11$ for Control, $n=6$ for Surgery-Vehicle, $n=7$ for SurgerySRT1720. One-way ANOVA with Tukey post-hoc test. b. Escape latency in training trials of Morris water maze. $n=12$ for Control and Surgery-Vehicle, $n=9$ for Surgery-SRT1720. Two-way ANOVA with Tukey post-hoc test. c. Percentage of time spent in target quadrant in probe trials on the first and third after surgery. $\mathrm{n}=12$ for Control and Surgery-Vehicle, $\mathrm{n}=9$ for Surgery-SRT1720. Two-way ANOVA with Tukey post-hoc test $d$. Crossing times of the platform area in probe trials on the first and third after surgery. $n=$ 12 for Control and Surgery-Vehicle, $n=9$ for Surgery-SRT1720. Two-way ANOVA with Bonferroni post-hoc test. All data are shown as mean \pm SEM. n.s. not significant; ${ }^{\star} p<0.05$; ${ }^{* \star} p<0.01$. POD, postoperative day. 